S2 File. List of domestic violence support organizations/networks closed to the study sites

The research team referred the abused respondents seeking help to the following organizations:

\begin{tabular}{|c|c|}
\hline প্রতিষ্ঠানের নাম, ঠিকানা ও ফোন নম্বর & প্রদত্ত সেবাসমূহ \\
\hline ১. নারী ও শিশ্ট নির্যাতন প্রতিরোধ হেল্পলাইন সেন্টার: ১০৯ & $\begin{array}{l}\text { • নির্যাতনের শিকার নারী/শিশু, আত্নীয় বা } \\
\text { প্রতিবেশী ফোন করে নির্যাতনের কথা }\end{array}$ \\
\hline ২. বাংলাদেশ পুলিশের জরুরিসেবা / হেল্পলাইন নম্বর: ৯৯৯ & $\begin{array}{l}\text { হয় এবং দ্রুত উদ্ধার-ঢীম পাঠানো হয়। } \\
\text { - নিরাপত্তা ও সকল লেবার ব্যাবস্থা করা হয়। }\end{array}$ \\
\hline $\begin{array}{l}\text { ৩. ওয়ান স্টপ ক্রাইসিস সেন্টার/সেলু ওসিসি * } \\
\text { রাজশাহী নেডিকেল কলেজ হাসপাতাল,' নোবাইল: ০১৭১৩ ৩৬৬৬৩৭ } \\
\text { টাঙ্গাইল মেডিকেল কলেজ হাসপাতাল, ফোন: ০১৭৪৫ ৪৭৭৭১১, ০১৭৩০ ৭b১০০৫ }\end{array}$ & $\begin{array}{ll}\bullet & \text { বিনামূল্যে চিকিৎসা সেবা } \\
\bullet & \text { মানসিক সমর্থন ও উপদেশ } \\
\bullet & \text { মধ্যস্থতা ও বিরোধ নিস্পক্তি } \\
\bullet & \text { বিনামুল্যে আইনগত সহায়তা } \\
\bullet & \text { পুলিশি সহায়তা/নিরাপত্তা প্রদান } \\
\bullet & \text { নারী/শিশুকে নিরাপদ আশ্রয় প্রদান }\end{array}$ \\
\hline $\begin{array}{l}\text { 8. উপজেলা মহিলা বিষয়ক কর্মকর্তার অফিস * } \\
\text { রাজশাহী, দুগাপুর : দোন: ০১৭১৭-৩৩৯৭৪৯, ০১৭৬২-৮-৬৫৬১৬ } \\
\text { রাজশাহী, গোদাগাড়ী (উপজেলা পরিষদ): ফোন: ০২৪৭- ৮-৫২২৮- } \\
\text { টাঙ্গাইল, মধুপুর (উপজেলা পরিষদ): ০১৭১৫৪০৪৭৭৫, ০১৭৬৬৯১৬৩৬ }\end{array}$ & $\begin{array}{l}\text { • মধ্যস্থতা ও বিরোধ নিস্পত্তি } \\
\text { • বিনামূল্যে আইনগত সহায়তা } \\
\text { • প্রশিক্ষণ ও পুনর্বাসনে সহায়তা }\end{array}$ \\
\hline $\begin{array}{l}\text { ৫. ব্র্যাক } \\
\text { রাজশাহী: (গোদাগাড়ী, সি এন বি মোড়), মোবাইল: ০১৭০০-৭৯৬৫৩০ } \\
\text { রাজশাহী: (দুর্গাপুর, সিংগা, ,নোবাইল: ০১৭০০-৭৯৬৫২৮ } \\
\text { টাঙ্গাইল: (মধুপুর, ময়মনসিংহ রোড), মোবাইল: ০১৭০০-৭৯৬৪২৫ }\end{array}$ & $\begin{array}{l}\text { • } \quad \text { মধ্যস্থতা ও বিরোধ নিস্পত্তি } \\
\text { • } \quad \text { সাইকো-নোশাল কাউন্সিলিং } \\
\text { • আইনগত সহায়তা }\end{array}$ \\
\hline $\begin{array}{l}\text { ৬. বাংলাদেশ মহিলা পরিষদ * } \\
\text { রাজশাইী (রাণীবাজার), নোবাইল:: ০১৭১২-৮-০৩৮-৮০ } \\
\text { টাঙ্গাইল অফিস (টাঙ্গাইল), নোবাইল: ০১৭১৯-৭৮-৬১১ }\end{array}$ & $\begin{array}{l}\text { • } \text { মধ্যস্থতা ও বিরোধ নিস্পত্তি } \\
\text { • বিনামূল্যে আইনগত সহায়তা } \\
\text { • নারী/শিশুকে নিরাপদ আশ্রয় প্রদান }\end{array}$ \\
\hline $\begin{array}{l}\text { ৭. বাংলাদেশ লিগ্যাল এইড এবং সেবা ট্রাস্ট (ব্লাস্ট) * } \\
\text { রাজশাই: বার এলোসিয়েশন নিউ ভবন, ফোন: ০২৪৭-b-১১(ৃ৩০, ০১৭৯১-৬৯৪৪৭৯ } \\
\text { টাঙ্গাইল: রোকেয়া মঞ্জিল, কেডিকেল কলেজ রোড, ফোন: ০৯২১-৬২২০৭ }\end{array}$ & $\begin{array}{l}\text { • } \text { মধ্যস্থতা ও বিরোধ নিস্পত্তি } \\
\text { • বিনামূন্যে আইনগত সহায়তা } \\
\text { • ুলিশি সহায়তা/নিরাপত্তা প্রদান }\end{array}$ \\
\hline $\begin{array}{l}\text { b. বাংলাদেশ জাতীয় মহিলা আইনজীবী সমিতি * } \\
\text { রাজশাইী: মাছুয়া পাড়ার গলি, শিরোইল, নোবাইল: ০১৭১১-০৭৫০৬৮- } \\
\text { টাঙ্গাইল: বার অফিসের পশ্চিম পাশ, মোবাইল: ০১৮-১b--8৪৩২৯১ }\end{array}$ & $\begin{array}{l}\text { • } \text { মধ্যস্থতা ও বিরোধ নিস্পত্তি } \\
\text { • বিনামূল্যে আইনগত সহায়তা } \\
\text { • নারী/শিশুকে নিরাপদ আশ্রয় প্রদান }\end{array}$ \\
\hline $\begin{array}{l}\text { ৯. নারী নির্যাতন প্রতিরোধ সেল * } \\
\text { রাজশাহী: মহিলা বিষয়ক অধিদপ্রুর (উপ-পরিচালকের কার্যালয়), } \\
\text { তেরখাদিয়া, ঢোন: ০৭২১-৭৬১৭৬, ০১৭১৪-২২৯৬৬৬ } \\
\text { টাঙ্গাইল: মহিলা বিষয়ক অধিদপ্ত্র, নোবাইল: ০১৭১৫ ৬০৯১৭৬ }\end{array}$ & $\begin{array}{l}\text { • বিনামূল্যে আইনগত সহায়তা } \\
\text { • প্রশিক্ষণ ও পুনর্বাসনে সহায়তা }\end{array}$ \\
\hline $\begin{array}{l}\text { ১০. মহিলা সহায়তা কেন্দ্র কর্মসূচি * } \\
\text { রাজশাহী: বহরমপুর শেষ মাথার নোড়, তেরখাদিয়া পশ্চিম দিক } \\
\text { ঢোন: ০৭২১-৭৬১০৬৫, ০১৯১-৬৬৯৬৯৫ }\end{array}$ & $\begin{array}{l}\text { • } \text { মধ্যস্থতা ও বিরোধ নিস্পত্তি } \\
\bullet \quad \text { বিনামূল্যে আইনগত সহায়তা } \\
\text { • } \quad \text { নারী/শিশ্ডকে নিরাপদ আশ্রয় প্রদান }\end{array}$ \\
\hline $\begin{array}{l}\text { ১১. জেলা লিগ্যাল এইড অফিস * } \\
\text { রাজশাহী, ফোন: ০৭২১- ৭৭১৩৭৩, ০১৭০০-৯৭৯৮৯০, ০১৭০০-৭b-৪২b-b- } \\
\text { টাঙ্গাইল অফিস (টাঙ্গাইল), ফোন: ০৯২১-৬১৬৩৭ }\end{array}$ & • বিনামূল্যে আইনগত সহায়তা \\
\hline $\begin{array}{l}\text { ১২. এসোসিয়েশন ফর কমিউনিটি ডেভেলপমেন্ট (এসিডি) * } \\
\text { এইচ-৪১, সাগরপাড়া,৬১০০,রাজশাইী। } \\
\text { ঢোন: ০৭২১- ৭৭০৬০, ০১৭১৬-৬৮-২০৯০ }\end{array}$ & 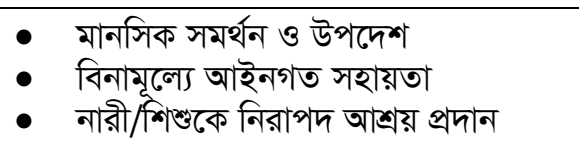 \\
\hline $\begin{array}{l}\text { ১৩. সচেতন * } \\
\text { পলিটেকনিক ইন্সটিটিউট এর পশ্চিনে,সপুরা, রাজশাহী, ফোন: ০১৭৯৩- ০৪০২৭০ }\end{array}$ & • বিনামূল্যে আইনগত সহায়তা \\
\hline $\begin{array}{l}\text { ১8. পারিবার ও শিশু কল্যাণ কেন্দ্র * } \\
\text { মবুপুর: মুক্তিযুদ্ধ অফিলের সামনে ,ময়মনসিংহ রোড, মোবাইল: ০১৭১৩৫৬০৫৮-৩ }\end{array}$ & $\begin{array}{l}\text { • } \quad \text { মধ্যস্থতা ও বিরোধ নিস্পত্তি } \\
\text { • } \quad \text { পরামর্শ ও আইনগত সহায়তা }\end{array}$ \\
\hline
\end{tabular}

*The team had pre-study arrangements with these organizations regarding their referral services 


\section{English Version}

The research team referred the abused respondents seeking help to the following organizations:

\begin{tabular}{|c|c|}
\hline Name of the organization & Given Services \\
\hline $\begin{array}{l}\text { National Helpline Centre for Violence against } \\
\text { Women and Children- } 109\end{array}$ & $\begin{array}{l}\text { Victim women and children, parents, relatives or neighbor can } \\
\text { call to this toll free phone numbers. } \\
\text { A law-enforcement team/police take immediate action. } \\
\text { - Immediately rescue violence victim women and children and help them. }\end{array}$ \\
\hline $\begin{array}{l}\text { One Stop Crisis Center (OCC) } \\
\text { Rajshahi Medical College Hospital, } \\
\text { Mobi:01713-366637 } \\
\text { Tangail Medical College Hospital, } \\
\text { Phn:01745-477711,01730-781005 }\end{array}$ & $\begin{array}{ll}- & \text { Free medical service } \\
- & \text { Mental support and advice } \\
- & \text { Arbitration and dispute mitigation. } \\
- & \text { Police protection and help; Free Legal Service } \\
- & \text { Provide secure shelter to victim women and children. }\end{array}$ \\
\hline $\begin{array}{l}\text { Upzilla Women Affairs Office } \\
\text { Rajshahi (Durgapur): Phn: 01717-339749,01762- } \\
\text { 865616; Rajshahi (Godagari), Phn:0247-856278; } \\
\text { Tangail (Modhupur), Phn: 01715-404775 } \\
\end{array}$ & $\begin{array}{ll}- & \text { Free legal aid. } \\
- & \text { Arbitration and dispute mitigation. } \\
- & \text { Help in rehabilitation and training. }\end{array}$ \\
\hline $\begin{array}{l}\text { BRAC } \\
\text { Rajshahi (Godaghari), Mob:01700-796530 } \\
\text { Rajshahi(Durgapur), Phn: 01700-796528 } \\
\text { Tangail (Modhupur),Phn:01700- } 796425\end{array}$ & $\begin{array}{ll}- & \text { Arbitration and dispute mitigation. } \\
- & \text { legal aid } \\
- & \text { Psycho-social concealing. }\end{array}$ \\
\hline $\begin{array}{l}\text { Bangladesh Mohila Porishad } \\
\text { Rajshahi (Rani Bazar), Phn: 01712-803880 } \\
\text { Tangail, Phn: 01719-786611 } \\
\end{array}$ & $\begin{array}{ll} & \text { Arbitration and dispute mitigation. } \\
- & \text { Free legal aid } \\
- & \text { Provide secure shelter to women and children. }\end{array}$ \\
\hline $\begin{array}{l}\text { Bangladesh Legal Aid and Service Trust } \\
\text { (BLAST), Rajshahi (Bar Association New } \\
\text { Bhavan), Phn:0247-811533,01791694479, } \\
\text { Tangail ( Rokeya Monjil, Medical College } \\
\text { Road), Phn:0921-62207 }\end{array}$ & $\begin{array}{ll}- & \text { Arbitration and dispute mitigation. } \\
- & \text { Free legal aid } \\
- & \text { Police protection/security. }\end{array}$ \\
\hline $\begin{array}{l}\text { Bangladesh National Women Lawyers' } \\
\text { Association, Rajshahi :( Shiroil) Phn: 01711- } \\
\text { 075068, Tangail ( Bar Office), Phn:01818443291 }\end{array}$ & $\begin{array}{ll} & \text { Arbitration and dispute mitigation. } \\
- & \text { Free legal aid } \\
- & \text { Provide secure shelter to women and children. }\end{array}$ \\
\hline $\begin{array}{l}\text { Cell for the prevention of violence against } \\
\text { women, Department of women affairs } \\
\text { Rajshahi, Phn: 0721-761736, 01714-229666 } \\
\text { Tangail, Phn: } 01715-609176 \\
\end{array}$ & $\begin{array}{ll}- & \text { Free legal aid } \\
- & \text { Help in rehabilitation and training. }\end{array}$ \\
\hline $\begin{array}{l}\text { Women's Help Center Programme } \\
\text { Ministry of women's Affairs, Rajshahi, Mob: } \\
\text { 0721-761065, 01916-690695 }\end{array}$ & $\begin{array}{ll} & \text { Arbitration and dispute solving. } \\
- & \text { Free legal aid } \\
- & \text { Provide secure shelter to women and children. }\end{array}$ \\
\hline $\begin{array}{l}\text { District Legal Aid Office } \\
\text { Rajshahi, phn:0721-771373, 01700- } \\
\text { 784288,01700-979890 } \\
\text { Tangail, phn: 0921-61637 }\end{array}$ & - $\quad$ Free legal aid \\
\hline $\begin{array}{l}\text { Association for Community Development } \\
\text { (ACD), } \\
\text { Rajshahi, Phn: 0721-770660, 01716-682090 }\end{array}$ & $\begin{array}{ll}- & \text { Mental support and advice } \\
- & \text { Free legal aid } \\
- & \text { Provide secure shelter to women and children. }\end{array}$ \\
\hline $\begin{array}{l}\text { Socheton } \\
\text { Rajshahi, Phn:01793-040270 }\end{array}$ & - $\quad$ Free legal aid. \\
\hline $\begin{array}{l}\text { Family and Children Welfare Center } \\
\text { Tangail (Modhupur),Phn: } 01713560583\end{array}$ & $\begin{array}{l}\text { - } \quad \text { Arbitration and dispute mitigation. } \\
\text { - Legal aid and advice }\end{array}$ \\
\hline
\end{tabular}

